\title{
Trigger of Apoptosis in Adenocarcinoma Gastric Cell Line (AGS) by a Complex of Thiosemicarbazone and Copper Nanoparticles
}

\section{Mahsa Badrooh}

Islamic Azad University Rasht Branch

Faezeh Shokrollahi

Islamic Azad University Rasht Branch

Shaghayegh Javan

Islamic Azad University Sari Branch

Taraneh Ghasemipour

Islamic Azad University Rasht Branch

\section{Samira Rezaei Mojdehi}

University of Guilan

\section{Mahboubeh Jahani Sayyad Noveiri}

Islamic Azad University Tonekabon Branch

\section{Mohammad Hedayati}

Guilan University of Medical Sciences

Ali Salehzadeh ( $\sim$ Salehzadehmb@yahoo.com )

Islamic Azad University Rasht Branch https://orcid.org/0000-0003-4238-0999

\section{Research Article}

Keywords: Apoptosis, Caspase-3, AGS cells, Thiosemicarbazone

Posted Date: August 9th, 2021

DOI: https://doi.org/10.21203/rs.3.rs-750522/v1

License: (1) (1) This work is licensed under a Creative Commons Attribution 4.0 International License.

Read Full License

Version of Record: A version of this preprint was published at Molecular Biology Reports on January 10th, 2022. See the published version at https://doi.org/10.1007/s11033-021-07043-z. 


\section{Abstract}

Seeking novel anticancer agents with minimal side effects against gastric cancer is vitally important. Copper, as an important trace element, takes roles in different physiologic pathways. Also, there is a higher demand for copper in cancer cells than normal ones. Copper complexes containing a therapeutic ligand could be promising candidates for gastric cancer chemotherapy. In this work, copper oxide nanoparticles were synthesized, functionalized with glutamic acid (CuO@Glu) and conjugated with thiosemicarbazone (Cuo@Glu/TSC NPs). The NPs were characterized and their antiproliferative potential against AGS cancer cells was investigated using MTT, flow cytometry, Hoechst staining, and caspase 3 activation assays. The FT-IR results showed the proper binding of TSC to CuO@Glu NPs and crystallinity of the prepared NPs was confirmed by the XRD pattern. The EDX analysis confirmed the presence of $\mathrm{Cu}$, $\mathrm{N}, \mathrm{C}, \mathrm{O}$, and $\mathrm{S}$ elements and lack of impurities. The Hydrodynamic size and zeta potential of the Cuo@Glu/TSC NPs were 710 nm and 27.5 mv, respectively. The NPs had spherical shape and were in a size range of 20-39 nm in diameter. This work revealed that CuO@Glu/TSC NPs efficiently inhibited the proliferation of AGS cells with significantly lower IC50 value $(203 \mu \mathrm{g} / \mathrm{mL})$ than normal HEK293 cells $(\mathrm{IC} 50=435 \mu \mathrm{g} / \mathrm{mL})$. Flow cytometry and Hoechst staining obviously revealed apoptosis induction among CuO@Glu/TSC treated cells, and caspase-3 activity significantly increased by 1.4 folds. This study introduced CuO@Glu/TSC as an efficient anticancer against gastric cancer cells with lower toxicity toward normal cells which could be employed for cancer treatment after further characterization.

\section{Introduction}

Gastric cancer is a prevalent and deadly cancer, with about one million annual new cases, in the world [1]. The disease is highly prevalent around the world especially in eastern and central Asian countries. Gastric cancer causes 783.000 deaths annually, which accounts for $8.3 \%$ of cancer-associated death [1]. Traditional cancer therapy including chemotherapy, radiotherapy, and surgery are expensive, not always efficient and have sometimes considerable side effects [2]. Therefore, finding novel and inexpensive anticancer agents with minimal side effects is crucially important to treat gastric cancer.

Upon introduction of cisplatin, as a novel and efficient anticancer agent, many efforts have been focused on finding metal-containing drugs [3]. Among metal elements, copper complexes have gained more attention due to their fewer side effects and toxicity. Copper is considered an important trace element in the body which is important for the proper activity of cellular proteins and enzymes [4]. Owing to the higher proliferation rate and physiological activity, there is a higher demand for copper in cancer cells in comparison with normal ones [5]. Therefore, due to the increased copper uptake by cancer cells, the preparation of antiproliferative copper complexes might provide selective toxicity against cancer cells.

Several copper complexes with anticancer activity have been reported [3, 6-7]. Among them, the complexes containing therapeutic ligands such as thiosemicarbazones (TSC) have been introduced as novel and efficient anticancer agents [3]. Thiosemicarbazide is able to interact with a variety of metals and to enhance their chemical activity. The anticancer potential metal NPs in conjugation with 
thiosemicarbazide was reported in the literature. Owing to the versatile coordination to metal ions and also, broad pharmaceutical potentials, including antibacterial, antiviral, and anti-tumor activities, TSC derivatives have been explored to prepare metal-TSC complexes, to be used as pharmaceutical agents [3, 6-7]. Several Copper/TSC complexes have been evaluated against a variety of cancer cells including colon adenocarcinoma HT-29, breast cancer cells, lymphoblastic leukemia, and cervical carcinoma $[3,6]$.

Many attempts have been performed to improve the hydrophobicity, anticancer potential, and stability of Copper/TSC complexes and also to minimize their side effects. The stable Copper/TSC complexes are able to reach the desired targets more efficiently and avoid nonselective interactions of the drug with different parts of cells [3]. Modification of TSCs framework to reduce the toxicity of Copper/TSC complexes toward normal cells was the goal of many studies [3, 7-8]. Therefore, in this work we aimed to synthesize copper oxide NPs, functionalize them with glutamic acid (CuO@Glu), and conjugate with TSC (CuO@Glu/TSC NPs). Then, the anticancer effect of CuO@Glu/TSC NPs on AGS cells was evaluated.

\section{Materials And Methods}

\section{Preparation of CuO and CuO@Glu/TSC NPs}

Preparation of CuO NPs was performed by co-precipitation method, as described elsewhere [9]. Briefly, $300 \mathrm{mg}$ of $\mathrm{CuCl}_{2} \cdot 2 \mathrm{H}_{2} \mathrm{O}$ was dissolved in $300 \mathrm{~mL}$ of distilled water; the $\mathrm{pH}$ was adjusted to 11 and the mixture was stirred at $80^{\circ} \mathrm{C}$ for two hours. Next, the CuO NPs were centrifuged, washed with distilled water, and dried at $70{ }^{\circ} \mathrm{C}$. A similar protocole was used for the synthesis of Cuo@Glu, but $150 \mathrm{mg}$ of Lglutamic acid was also added to the reaction mixture.

To synthesizeCuO@Glu/TSC, 300 mg of CuO@Glu and 200 mg of thiosemicarbazone were dissolved in $300 \mathrm{ml}$ of ethanol and stirred at $40^{\circ} \mathrm{C}$ for one hour. Then, the mixture was centrifuged; and the resulting NPs were harvested, washed with distilled water, and dried at $70^{\circ} \mathrm{C}$.

\section{Characterization ofCuO@Glu/TSCNPs}

X-ray diffraction (XRD) pattern of the CuO@Glu/TSC NPs was determined by a Philips X'Pert MPD diffractometer (Co-Ka X-radiation, $\mathrm{k}=1.79 \AA$ A), and dynamic light scattering (DLS), and Zeta potential analyses were used to characterize the stability and agglomeration state of NPs using a Zeta-sizer instrument (Ver. 6.32, Malvern Instruments Ltd). Also, the morphology, distribution, and size of the prepared CuO@Glu/TSC NPs were determined by scanning electron microscopy (SEM) (KYKY-EM3200) and transmission electron microscopy (TEM) (EM10C). Moreover, to analyze the composition of NPs was energy dispersive X-Ray (EDX) was employed using an X-ray diffraction spectrometer (Delta series KEVEX). Also, a Nicolet IR 100 spectrophotometer was employed to analyze the Fourier-transform infrared (FT-IR) spectra of the NPs. 


\section{Cell culture}

The adenocarcinoma gastric (AGS) and the human embryonic kidney 293 (HEK293) cells were received from the Pasteur Institute of Iran. The cell culture was performed in $25 \mathrm{~cm}^{2}$ cell culture flasks using the Dulbecco's modified Eagle (DMEM) medium supplemented with penicillin/streptomycin $(100 \mathrm{U} / \mathrm{mL})$ and $100 \%$ fetal bovine serum (FBS) (Gibco, Netherland). To prepare cell monolayers, the incubation was performed at $37^{\circ} \mathrm{C}$ with $5 \% \mathrm{CO}_{2}$.

\section{Evaluation of the viability of cells treated with CuO@Glu/TSCNPs}

The antiproliferative potential of CuO@Glu/TSC NPs against the AGS (cancer cell line) and HEK293 cells (as normal human cells) was investigated using the 2-(4,5-dimethythiazol-2-yl) -2,5-diphenyltetrazolium bromide (MTT) assay. Cisplatin was also used as a control drug against AGS cells. The assay was performed in triplicates.

At first, the cell monolayers with a cell population of $1 \times 10^{4}$ cell/well were prepared in microtiter plates. The cells were exposed to CuO@Glu/TSC NPs at different concentrations in a range of $0-500 \mu \mathrm{g} / \mathrm{mL}$ for $24 \mathrm{~h}$. As a positive control, similar concentrations of cisplatin were also used against the AGS cells. After that, the MTT solution $(0.5 \mathrm{mg} / \mathrm{mL})$ was added to each well and the plate was incubated for 4 hours. After the incubation period, the medium was removed and $200 \mu \mathrm{L}$ of dimethyl sulfoxide was added to the wells, and the plate was shaken for $30 \mathrm{~min}$. Finally, the optical absorption of the wells was determined at $590 \mathrm{~nm}$ using a microplate reader (Bio-Rad, Hercules, CA, USA). The following formula was used to determine the $50 \%$ inhibition concentration (IC50) values of CuO@Glu/TSC NPs and cisplatin against the studied cells [10]:

$$
\text { Inhibition }(\%)=\frac{\text { Abs of control-Abs of Test }}{\text { Abs of control }} \times 100
$$

\section{Flow cytometry assay}

Flow cytometry analysis was performed to determine the apoptosis/necrosis rate of the AGS cells treated with CuO@Glu/TSC NPs using a flow cytometry kit (Roche, Germany). The AGS cells were treated with the NPs at IC ${ }_{50}$ concentration and incubated for $24 \mathrm{~h}$. Then, the cells were harvested and washed with phosphate-buffered saline (PBS). Then, $10 \mu \mathrm{L}$ of Annexin-V FLUOS and $5 \mu \mathrm{L}$ of propidium iodide (PI) were added to the cells and the population of apoptotic/necrotic cells was determined by a flow cytometry device (Partec, Germany). 


\section{Hoechst 33258 staining}

The nuclear damages caused by CuO@Glu/TSC NPs in treated AGS cells were evaluated by the Hoechst staining method [11]. At first, the AGS cells $\left(4 \times 10^{5}\right.$ cells/well) were prepared and treated with CuO@Glu/TSC NPs at IC ${ }_{50}$ concentration. The AGS cells without NPs exposure were regarded as control cells. After $24 \mathrm{~h}$ incubation, the cells were stained with Hoechst 33258 dye and visualized using a fluorescent microscope (Incell Analyser 2000, USA).

\section{Caspase-3 activity}

The activity of caspase-3 among the AGS cells, treated with CuO@Glu/TSC NPs and also control cells was evaluated using a colorimetric assay kit (Sigma-Aldrich, CASP3C). In brief, the AGS cells with a population of $5 \times 10^{6}$ cells/well were prepared in 6-well plates and treated with CuO@Glu/TSC NPs at $\mathrm{IC}_{50}$ concentration for $24 \mathrm{~h}$. Then, the cells were harvested, lysed using a cell lysis buffer, and centrifuged. Finally, DEVD-pNA was added to the cell supernatant and the caspase-3 activity was determined by measuring the absorbance of $p$-nitroaniline $(\mu \mathrm{M})$ released from the substrate at $405 \mathrm{~nm}$ [12].

\section{Statistical analysis}

The cellular toxicity assays were conducted in triplicates and the results were presented as mean \pm SD. Statistical analyses were performed using SPSS. 16.0 software (SPSS Inc., Chicago, IL). The cellular toxicity of the compounds was expressed as mean $\pm S D$, and the statistical significance of data was analyzed by one-way ANOVA. The p-value less than 0.05 was considered as statistically significant.

\section{Results And Discussion}

\section{Characterization of CuO@Glu/TSC NPs}

The X-ray diffraction (XRD) pattern for the CuO@Glu/TSC nanoparticles was displayed in Figure 1. The XRD pattern for CuO@Glu/TSC showed the typical reflection peaks at $2 \theta$ values of $32.3^{\circ}, 38.6^{\circ}, 48.7^{\circ}$, 58.1. $0^{\circ}$ and $68.0^{\circ}$ corresponding to (110), (111), (202), (202) and (220) planes of CuO nanoparticles. The reflections could be indexed to face-centered cubic phase of $\mathrm{CuO}$, in accordance with the JCPDS card no. 80-1268 [13]. The sharpness and the intensity of the peaks imply the high crystallinity of the CuO@Glu/TSC NPs.

The FT-IR spectra of CuO@Glu and CuO@Glu/TSC NPs in the range of $400 \mathrm{~cm}^{-1}$ to $4000 \mathrm{~cm}^{-1}$ were presented in Figure 2. There are two sharp absorption peaks at $427 \mathrm{~cm}^{-1}$ and $535 \mathrm{~cm}^{-1}$ that are corresponding to the stretching vibrations of metal- 0 bonds supporting the presence of the monoclinic 
phase of copper oxide NPs [14]. The band at $1607 \mathrm{~cm}^{-1}$ corresponds to the $\mathrm{C}=\mathrm{O}$ vibration of $-\mathrm{COOH}$ groups for glutamic acid on the surface of CuO NPs.

The band at $3392 \mathrm{~cm}-1$ corresponding to the $\mathrm{O}-\mathrm{H}$ stretching vibrations show the vibrational modes of water molecules adsorbed on the surface of the NPs. Also, the peak at around $1955 \mathrm{~cm}-1$ in FT-IR spectrum of CuO@Glu/TSC is associated with the O-H bending. The comparison of FT-IR spectra of synthesized CuO@Glu and CuO@Glu/TSC confirms the successful conjugation of thiosemicarbazide compound on the surface of CuO@Glu NPs. The strong band observed at $1339 \mathrm{~cm}-1$ in the spectrum of CuO@Glu/TSC can be devoted to the v (C-N) stretching of thiosemicarbazone in the synthesized nanoparticles. The comparison of FT-IR spectra of the CuO@Glu NPs and synthesized CuO@Glu/TSC confirms the successful conjugation of this organic compound on the surface of CuO@Glu NPs. For instance, the band around $800 \mathrm{~cm}-1$ in the IR spectrum is corresponding to $v(C=S)$ stretching $[11,14]$.

The morphology and size of the CuO@Glu/TSC NPs were evaluated by scanning and transmission electron microscopy. The particles were approximately spherical with the particle size in a range of 20-39 nm. Figures 3 and 4 display the SEM and TEM images of the CuO@Glu/TSC NPs.

Energy dispersive X-Ray (EDX) was employed to analyze the Elemental composition of CuO@Glu/TSC NPs. The EDX analysis showed strong signals for the presence of $\mathrm{Cu}$ element, and also signals for the $\mathrm{C}$, $\mathrm{O}, \mathrm{N}$, and $\mathrm{S}$ atoms. The presence of no other EDX signal clearly indicates the purity of the synthesized NPs (Figure 5, Table 1). Moreover, the hydrodynamic size and zeta potential of CuO@Glu/TSC NPs were measured $710 \mathrm{~nm}$ and $27.5 \mathrm{mv}$, respectively. The proper zeta potential of the CuO@Glu/TSC NPs could provide sufficient repulsive force between particles to avoid agglomeration of the NPs and provides satisfactory colloidal stability [15] (Figure 6).

Table 1

EDS analysis of CuO@Glu/TSC NPs

\begin{tabular}{|llllll|}
\hline Elements & $\mathrm{K}$ & $\mathrm{Kr}$ & Weight (\%) & Atomic (\%) & ZAF \\
\hline $\mathbf{C}$ & 0.0707 & 0.0357 & 17.9 & 31.72 & 0.1996 \\
\hline $\mathbf{N}$ & 0.0720 & 0.0364 & 17.2 & 26.14 & 0.2114 \\
\hline $\mathbf{O}$ & 0.0828 & 0.0418 & 17.1 & 22.78 & 0.2442 \\
\hline $\mathbf{S}$ & 0.1689 & 0.0853 & 10.2 & 6.78 & 0.8357 \\
\hline $\mathbf{C u}$ & 0.6055 & 0.3058 & 37.6 & 12.58 & 0.8140 \\
\hline Total & 1.0000 & 0.5050 & 100.00 & 100.00 & \\
\hline
\end{tabular}

\section{Cytotoxic effect of CuO@Glu/TSC NPs}


The anti-proliferative potential of CuO@Glu/TSC NPs against AGS and HEK293 cell lines was evaluated. A considerable anti-proliferative effect of CuO@Glu/TSC NPs against AGS cells was observed with IC50 value of $203 \mu \mathrm{g} / \mathrm{mL}$. Also, the prepared NPs showed a significantly less anti-proliferative effect on the HEK293, as normal human cells, with an IC50 value of $435 \mu \mathrm{g} / \mathrm{mL}$. However, the anticancer potential of the NPs against AGS cells was significantly less than cisplatin, which exhibited the IC50 value of $65 \mu \mathrm{g} / \mathrm{mL}$. The relative viability of the cells exposed to different concentrations of CuO@Glu/TSC NPs and cisplatin was presented in Figure 7.

As a trace element with specific functions inside the eukaryotic cells, this element has lower toxicity to normal human cells [3]. Also, due to the higher physiological state of cancer cells, they show a higher demand for this element, which could provide selective toxicity of copper-containing drugs against cancer cells $[3,6]$. Combination of copper with a variety of therapeutic ligands, including imidazoles and phosphines, as well as TSC derivatives showed a promising anticancer potential $[3,16]$.

Several anticancer mechanisms have been proposed to be mediated by copper. The anticancer potential of copper has been mainly associated with the oxidative stress caused by the production of reactive oxygen species (ROS) and also, Glutathione (GSH) oxidation $[3,6]$. The oxidative imbalance damages cellular molecules, including nucleic acids, lipids, and proteins, which triggers cell apoptosis [6, 17-18].

According to the literature, copper complexes have shown proteasome inhibitory potential in different cell lines and copper element had a crucial role in this issue. The proteasome, a large and multi-catalytic protease, is responsible for the degradation of damaged proteins and also regulating the concentrations of cell cycle regulatory proteins, including cyclins and p53 (144 and 145 from 4). Due to the higher load of copper inside cancer cells than normal cells, a higher proteasome inhibitory potential and thus, apoptosis induction by copper complexes was reported, which efficiently inhibited the proliferation of cancer cells [19-20].

Similar to other metal complexes, copper complexes can undergo redox reaction and ligand substitution which enable them to interact with a variety of biological substrates. Thus, conjugation of therapeutic ligands with copper could provide efficient cytotoxicity against cancer cells by the action of both partners $[3,6]$.

TSCs are considered as efficient cytotoxic agents, which supress cancer cells proliferation by inhibition of cellular ribonucleotide phosphate reductase, the enzyme necessary for DNA replication [6]. Also, it was reported that the cytotoxicity of copper complexes containing coordinating ligands, including TSCs could be associated to the binding of the ligands to cell DNA which inhibits genome replication and transcription, and finally results in cell apoptosis [6].

Based on the results and above considerations we found that the conjugation of TSC to copper oxide NPs could inhibit the proliferation of cancer cells and results in higher toxicity to AGS cells compared with normal human cells. In addition, the functionalization of CuO@Glu/TSC NPs using glutamic acid seems to improve their stability and bioavailability. In agreement with our finding, several studies aimed to 
increase the hydrophilicity of $\mathrm{Cu} / \mathrm{TSC}$ complexes by modification of TSCs framework to improve their stability and reported promising anticancer characteristics $[8,21]$.

Moreover, investigating the antitumor effect of copper-2-keto-3- ethoxybutyraldehydebis(thiosemicarbazone) (KTS) revealed that the KTS ligand could stabilize the oxidation state of ${ }^{+2}$ copper. Following uptake of the complex by cancer cells, the complex is dissociated by interaction with cellular thiols. This dissociation generates $\mathrm{Cu}(\mathrm{I})$, which may act as heavy metal poison $[6,22]$. The similar synergistic potential could be also hypothesized to other CuO-TSC complexes.

\section{Apoptosis induction of CuO@Glu/TSC NPs in AGS cells}

Figure 8 displays the apoptosis of CuO@Glu/TSC treated and control AGS cells. The results were presented in the diagrams with four regions where Q1 region displays cell necrosis, Q2 and Q3 represent late and early apoptosis, and Q4 for live cells. Our results showed that early apoptosis occurred among the majority of CuO@Glu/TSC treated cells (69\%) and $11.5 \%$ of cells experienced late apoptosis, which was significantly higher than control cells. In addition, $96.7 \%$ and $19.3 \%$ of control and NPs treated cells remained alive. Also, only $0.27 \%$ of cell necrosis was found among CuO@Glu/TSC treated cells. The apoptosis induction by copper NPs conjugated to the TSC derivatives against Burkitt's lymphoma cells has been reported previously, which is in agreement with our finding [22]. As described above, the apoptotic effect of CuO@Glu/TSC NPs could be associated with the DNA damages caused by oxidative stress, inhibition of DNA replication, and cellular proteasome inhibition $[3,6]$.

\section{Hoechst 33258 staining}

Hoechst staining was performed on CuO@Glu/TSC treated and control cells to evaluate the presence of compact chromatin of apoptotic nuclei. The apoptosis morphological characteristics, including condensed chromatin and chromatin fragmentation, and apoptotic bodies were found among the AGS cells treated with CuO@Glu/TSC NPs. In contrast, the control cells did not show considerable changes (Figure 9). As stated above, the increased ROS generation by CuO@Glu/TSC could results in DNA strand breakage and apoptosis induction [6]. The apoptotic morphological characteristics of CuO@Glu/TSC treated cells comply well with the results from the MTT and flow cytometry assays.

\section{Caspase 3 activity}

The activity of caspase-3 among CuO@Glu/TSC treated AGS cells and control cells was investigated. The results showed that treating AGS cells with the NPs resulted in the increased activation of caspase-3. In other words, among NPs treated cells the caspase-3 activity significantly increased by 1.4 folds in comparison with the control cells (Figure 10). Caspases play a critical role in triggering of apoptosis [23]. In this study a significantly increased activity of caspase-3 was noticed, confirming the apoptosis 
induction in AGS cells following exposure to CuO@Glu/TSC NPs. In agreement with our work, previous studies showed increased transcription and activation of caspase-3 in a variety of cancer cells following treatment with copper NPs or copper complexes $[4,6,11,23]$. Generation of ROS and subsequently damages to cellular DNA is considered a major signaling for triggering apoptosis pathway [23]. In our opinion, after exposure of AGS cells to CuO@Glu/TSC NPs, generation of ROS by copper and also inhibition of cell proteasome, which regulates the concentration of p53 proteins, are major causes of apoptosis induction following upregulation of caspase proteins. In agreement with this, previous works reported that the activation of p53 among cancer cells treated with copper NPs indirectly promotes caspase-3 activity [24-25].

\section{Conclusion}

In this study, CuO@Glu/TSC NPs were synthesized and their anti-proliferative effect on AGS gastric cancer cells was investigated. Our results showed that the prepared NPs efficiently inhibited the cancer cell proliferation with significantly less IC50 than normal HKE293 cells. Also, the NPs induced early apoptosis, chromatin fragmentation, and increased activity of caspase-3 in AGS cells, which describe the cytotoxic mechanism of CuO@Glu/TSC NPs. This work showed a promising anti-proliferative effect of CuO@Glu/TSC against AGS cells, to be used for gastric cancer chemotherapy after further characterizations.

\section{Declarations}

\section{Ethics approval}

Not applicable.

\section{Author contributions}

Conceptualization: MB and AS; Methodology: AS; Formal analysis and investigation: MB, SHJ, SRM, MJ and MH; Writing - Original Draft Preparation: AS; Editing: AS; Resources: MB, SHJ, SRM, MJ, TGH and MH; Supervision: AS.

\section{Acknowledgment}

We would like to appreciate Dr. Zeinab Moradi-Shoeili for collaborate on the interpretation of chemical spectra.

\section{Conflict of interest}

The authors declare that they have no conflict of interest. 


\section{Funding}

This research received no specific grant from any funding agency in the public, commercial, or not-forprofit sectors.

\section{References}

1. Rawla, P., \& Barsouk, A. (2019). Epidemiology of gastric cancer: global trends, risk factors and prevention. Przeglad gastroenterologiczny, 14(1), 26. Doi: 10.5114/pg.2018.80001

2. Nagajyothi, P. C., Muthuraman, P., Sreekanth, T. V. M., Kim, D. H., \& Shim, J. (2017). Green synthesis: in-vitro anticancer activity of copper oxide nanoparticles against human cervical carcinoma cells. Arabian journal of chemistry, 10(2), 215-225. Doi: 10.1016/j.arabjc.2016.01.011

3. Tisato, F., Marzano, C., Porchia, M., Pellei, M., \& Santini, C. (2010). Copper in diseases and treatments, and copper-based anticancer strategies. Medicinal research reviews, 30(4), 708749. DOI:10.1002/med.20174

4. Qi, J., Yao, Q., Tian, L., \& Wang, Y. (2018). Piperidylthiosemicarbazones Cu (II) complexes with a high anticancer activity by catalyzing hydrogen peroxide to degrade DNA and promote apoptosis. European journal of medicinal chemistry, 158, 853-862. Doi: 10.1016/j.ejmech.2018.09.034

5. Lovejoy, D. B., Jansson, P. J., Brunk, U. T., Wong, J., Ponka, P., \& Richardson, D. R. (2011). Antitumor activity of metal-chelating compound $\mathrm{Dp} 44 \mathrm{mT}$ is mediated by formation of a redox-active copper complex that accumulates in lysosomes. Cancer research, 71(17), 5871-5880. Doi: 10.1158/00085472.CAN-11-1218

6. Tardito, S., \& Marchio, L. (2009). Copper compounds in anticancer strategies. Current medicinal chemistry, 16(11), 1325-1348. Doi: 10.2174/092986709787846532

7. Ma, Z. Y., Shao, J., Bao, W. G., Qiang, Z. Y., \& Xu, J. Y. (2015). A thiosemicarbazone copper (II) complex as a potential anticancer agent. Journal of Coordination Chemistry, 68(2), 277-294. Doi: 10.1080/00958972.2014.979811

8. Scovill, J. P., Klayman, D. L., \& Franchino, C. F. (1982). 2-Acetylpyridine thiosemicarbazones. 4. Complexes with transition metals as antimalarial and antileukemic agents. Journal of Medicinal Chemistry, 25(10), 1261-1264. Doi:10.1021/jm00352a036

9. Rahnama, A., \& Gharagozlou, M. (2012). Preparation and properties of semiconductor CuO nanoparticles via a simple precipitation method at different reaction temperatures. Optical and Quantum Electronics, 44(6-7), 313-322. Doi: 10.1007/s11082-011-9540-1

10. Shandiz, S. A. S., Montazeri, A., Abdolhosseini, M., Shahrestani, S. H., Hedayati, M., Moradi-Shoeili, Z., \& Salehzadeh, A. (2018). Functionalization of Ag nanoparticles by glutamic acid and conjugation of Ag@ Glu by thiosemicarbazide enhances the apoptosis of human breast cancer MCF-7 cells. Journal of Cluster Science, 29(6), 1107-1114. Doi: 10.1007/s10876-018-1424-0. 
11. Jarestan, M., Khalatbari, K., Pouraei, A., Sadat Shandiz, S. A., Beigi, S., Hedayati, M., ... \& Salehzadeh, A. (2020). Preparation, characterization, and anticancer efficacy of novel cobalt oxide nanoparticles conjugated with thiosemicarbazide. 3 Biotech, 10, 1-9. Doi: 10.1007/s13205-020-02230-4

12. Bejarbaneh, M., Moradi-Shoeili, Z., Jalali, A., \& Salehzadeh, A. (2020). Synthesis of Cobalt Hydroxide Nano-flakes Functionalized with Glutamic Acid and Conjugated with Thiosemicarbazide for Anticancer Activities Against Human Breast Cancer Cells. Biological Trace Element Research, 1-11. Doi: 10.1007/s12011-020-02049-3

13. Khan, S., Ansari, A. A., Khan, A. A., Abdulla, M., Al-Obaid, O., \& Ahmad, R. (2017). In vitro evaluation of cytotoxicity, possible alteration of apoptotic regulatory proteins, and antibacterial activity of synthesized copper oxide nanoparticles. Colloids and surfaces B: biointerfaces, 153, 320-326. Doi: 10.1016/j.colsurfb.2017.03.005

14. Rehana, D., Mahendiran, D., Kumar, R. S., \& Rahiman, A. K. (2017). Evaluation of antioxidant and anticancer activity of copper oxide nanoparticles synthesized using medicinally important plant extracts. Biomedicine \& Pharmacotherapy, 89, 1067-1077. Doi:10.1016/j.biopha.2017.02.101

15. Habibi, A., Shandiz, S. A., Salehzadeh A., Moradi-Shoeili, Z. (2020). Novel pyridinecarboxaldehyde thiosemicarbazone conjugated magnetite nanoparticulates (MNPs) promote apoptosis in human lung cancer A549 cells. JBIC Journal of Biological Inorganic Chemistry, 25(1), 13-22. Doi: 10.1007/s00775-019-01728-4.

16. Marzano, C., Pellei, M., Tisato, F., \& Santini, C. (2009). Copper complexes as anticancer agents. AntiCancer Agents in Medicinal Chemistry (Formerly Current Medicinal Chemistry-Anti-Cancer Agents), 9(2), 185-211. Doi:10.2174/187152009787313837

17. Halliwell, B., \& Aruoma, O. I. (1991). DNA damage by oxygen-derived species Its mechanism and measurement in mammalian systems. FEBS letters, 281(1-2), 9-19. Doi: 10.1016/00145793(91)80347-6

18. Schnitzer, E., Pinchuk, I., \& Lichtenberg, D. (2007). Peroxidation of liposomal lipids. European Biophysics Journal, 36(4-5), 499-515. Doi:10.1007/s00249-007-0146-2

19. Lodish, H., Berk, A., Kaiser, C. A., Krieger, M., Scott, M. P., Bretscher, A., ... \& Matsudaira, P. (2008). Molecular cell biology. Macmillan.

20. Marques, A. J., Palanimurugan, R., Matias, A. C., Ramos, P. C., \& Dohmen, R. J. (2009). Catalytic mechanism and assembly of the proteasome. Chemical reviews, 109(4), 1509-1536. Doi: $10.1021 / \mathrm{cr} 8004857$

21. Weder, J. E., Dillon, C. T., Hambley, T. W., Kennedy, B. J., Lay, P. A., Biffin, J. R., ... \& Davies, N. M. (2002). Copper complexes of non-steroidal anti-inflammatory drugs: an opportunity yet to be realized. Coordination Chemistry Reviews, 232(1-2), 95-126. Doi: 10.1016/S0010-8545(02)00086-3

22. Easmon, J., Pürstinger, G., Heinisch, G., Roth, T., Fiebig, H. H., Holzer, W., ... \& Hofmann, J. (2001). Synthesis, cytotoxicity, and antitumor activity of copper (II) and iron (II) complexes of $4 \mathrm{~N}$-azabicyclo [3.2. 2] nonane thiosemicarbazones derived from acyl diazines. Journal of Medicinal Chemistry, 44(13), 2164-2171. Doi: 10.1021/jm000979z

Page $11 / 19$ 
23. Siddiqui, M. A., Alhadlaq, H. A., Ahmad, J., Al-Khedhairy, A. A., Musarrat, J., \& Ahamed, M. (2013). Copper oxide nanoparticles induced mitochondria mediated apoptosis in human hepatocarcinoma cells. PloS one, 8(8), e69534. doi:10.1371/journal.pone.0069534

24. Ahamed, M., Siddiqui, M. A., Akhtar, M. J., Ahmad, I., Pant, A. B., \& Alhadlaq, H. A. (2010). Genotoxic potential of copper oxide nanoparticles in human lung epithelial cells. Biochemical and biophysical research communications, 396(2), 578-583. Doi: 10.1016/j.bbrc.2010.04.156

25. Fuentes-Prior, P., \& Salvesen, G. S. (2004). The protein structures that shape caspase activity, specificity, activation and inhibition. Biochemical Journal, 384(2), 201-232. Doi:

10.1042/BJ20041142

Figures

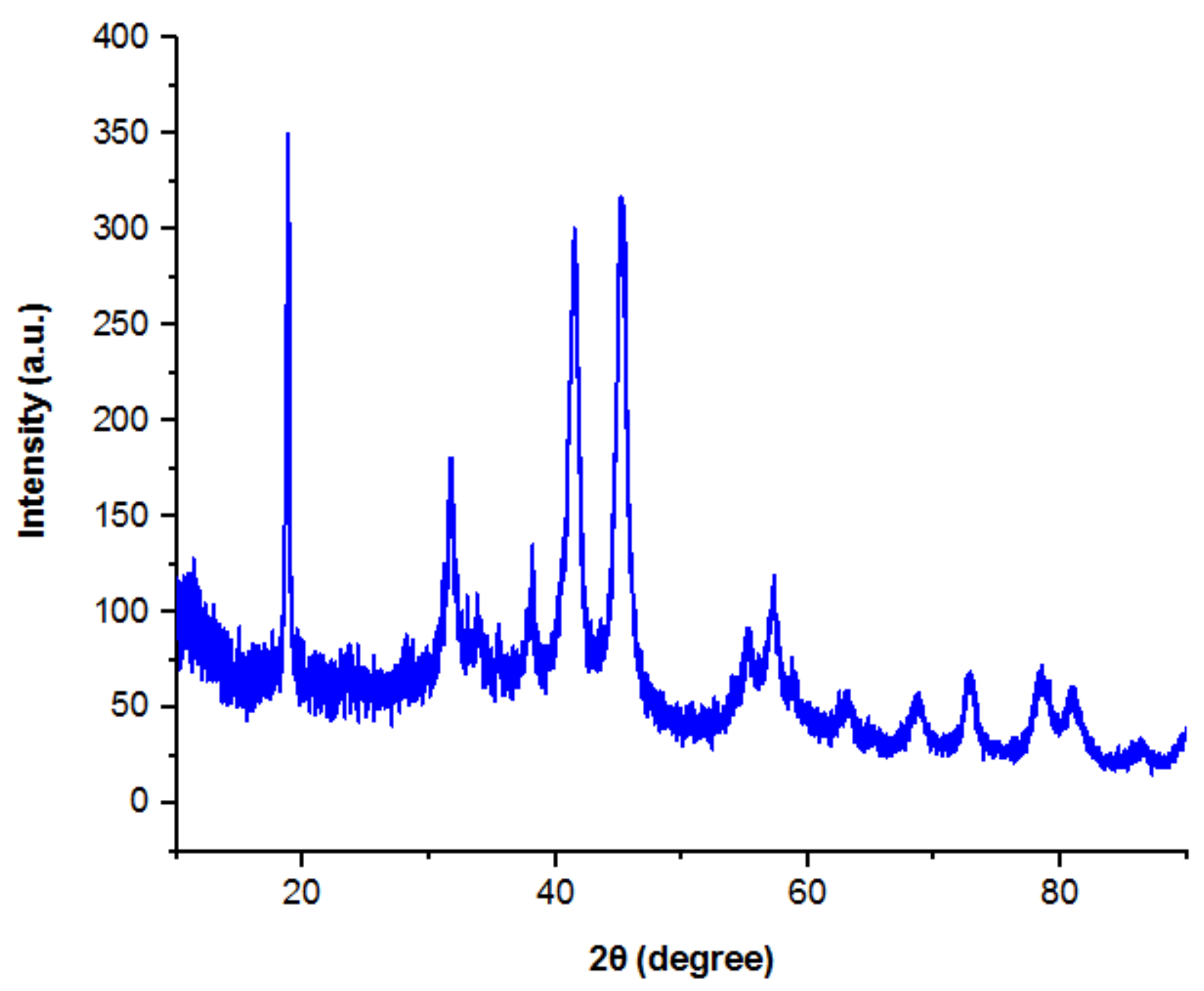

Figure 1

XRD pattern of the synthesized CuO@Glu/TSC NPs 


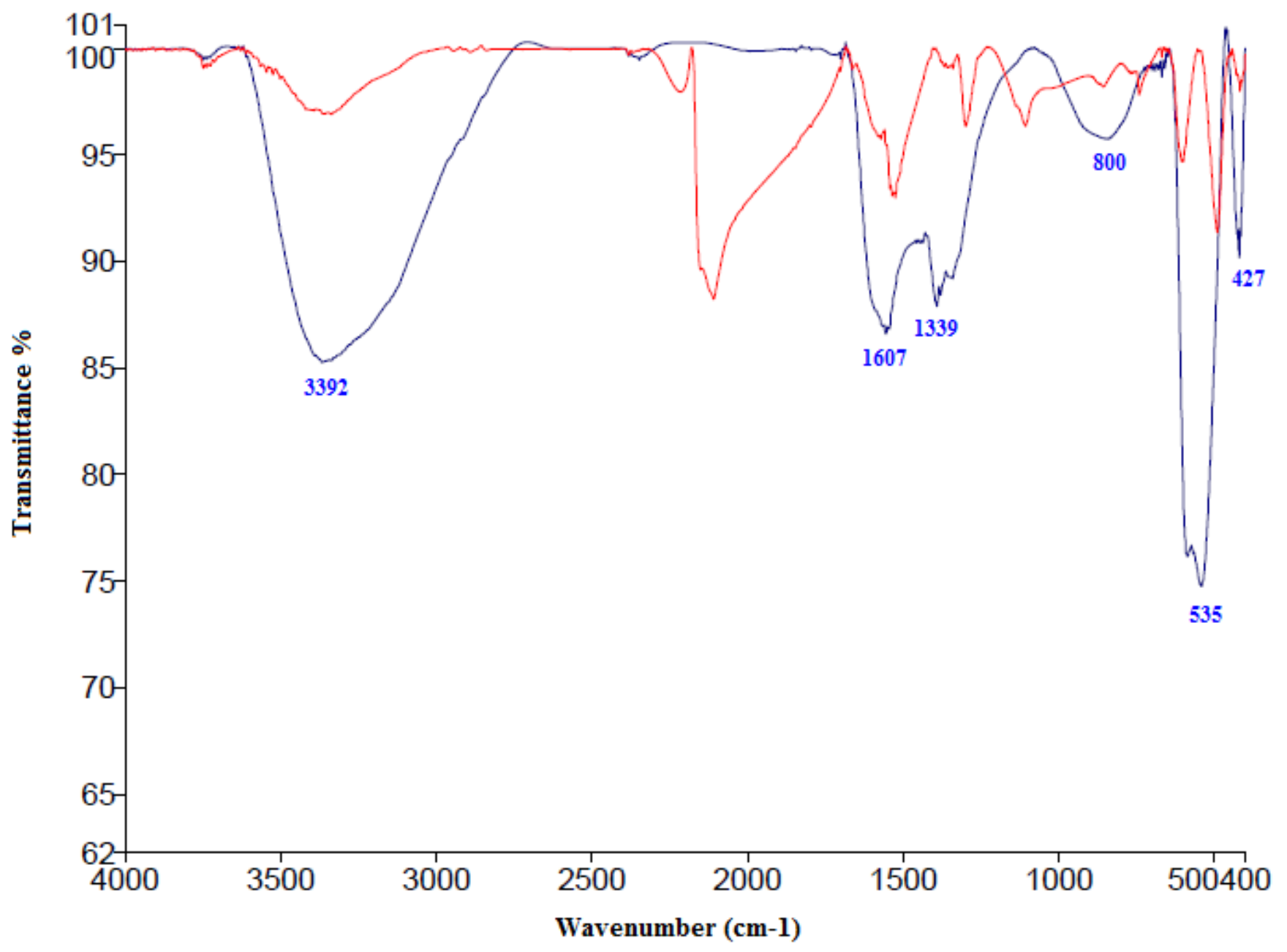

Figure 2

FT-IR spectra of CuO@Glu NPs (red graph),CuO@Glu/TSC NPs (blue graph) 

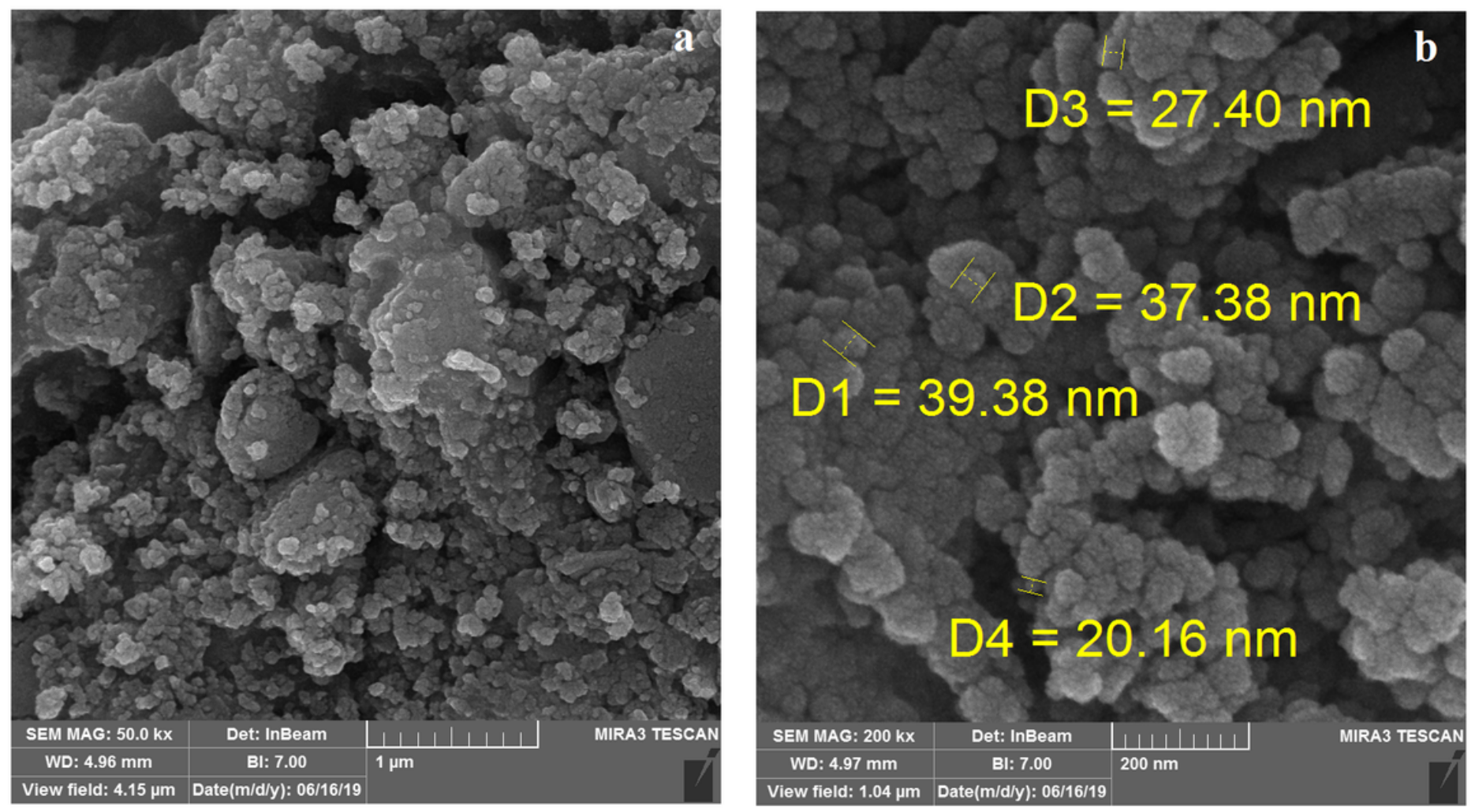

\section{Figure 3}

Scanning electron micrograph of the CuO@Glu/TSC NPs 


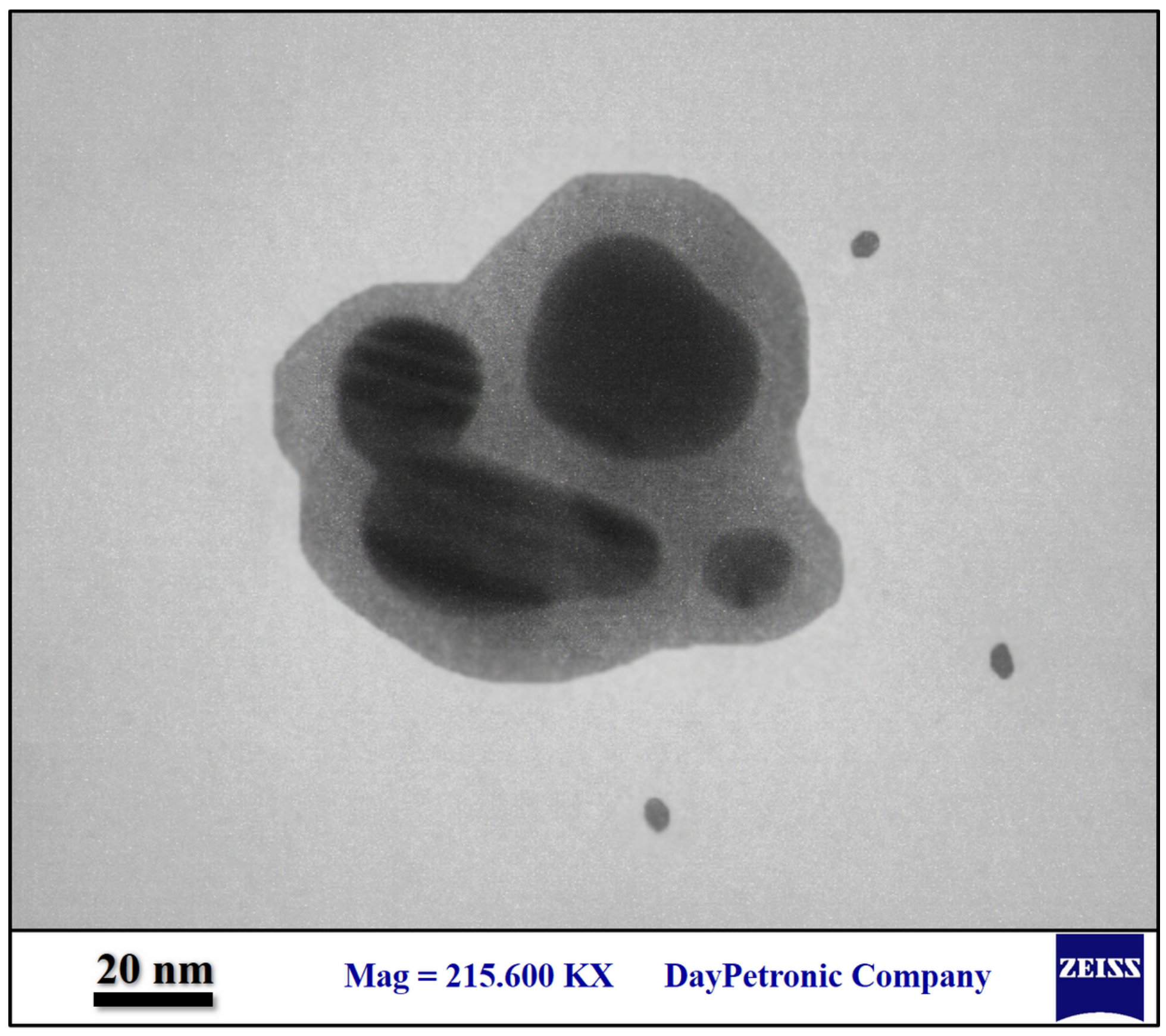

Figure 4

Transmission electron micrograph of the CuO@Glu/TSC NPs 


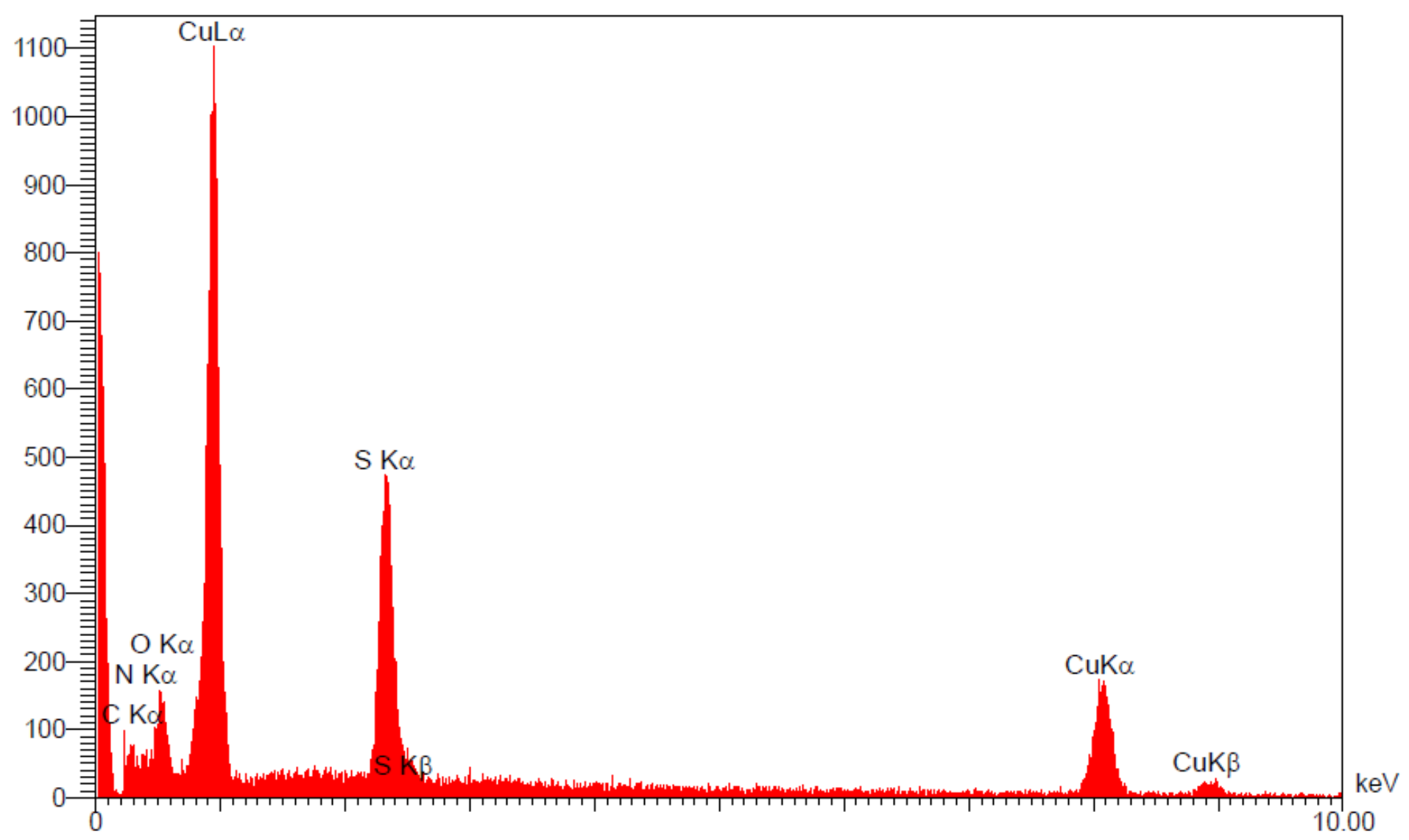

Figure 5

EDX analysis of synthesized CuO@Glu/TSC NPs
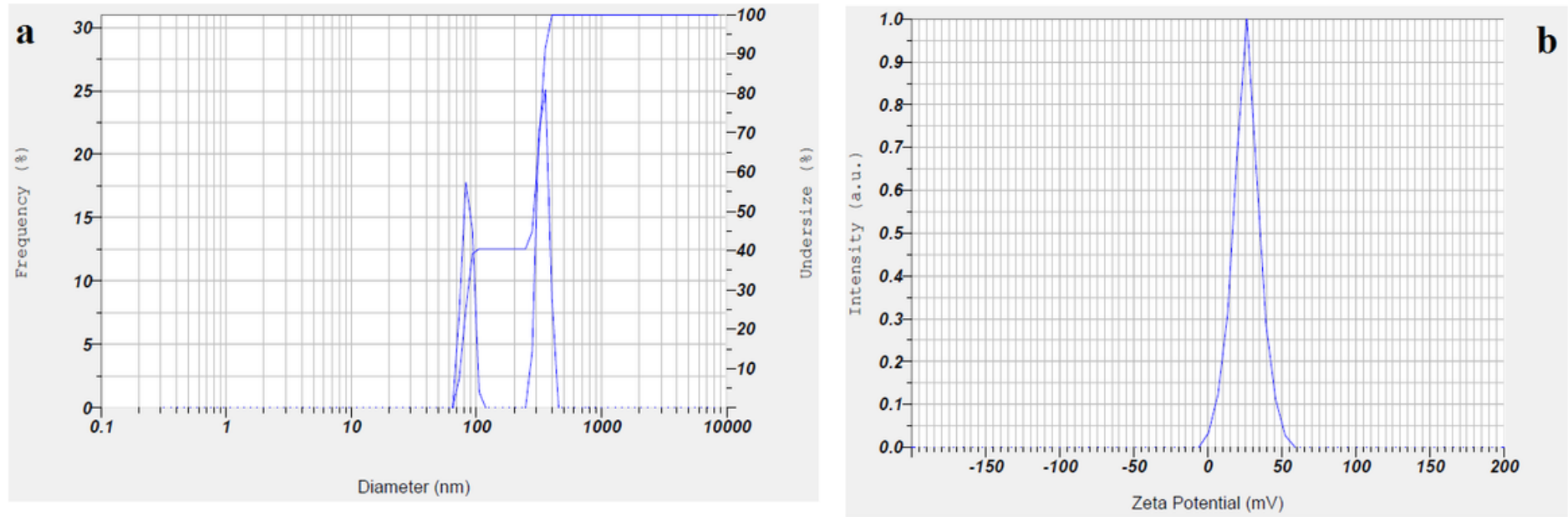

Figure 6

DLS (a) and Zeta potential (b) analyses of CuO@Glu/TSC NPs 

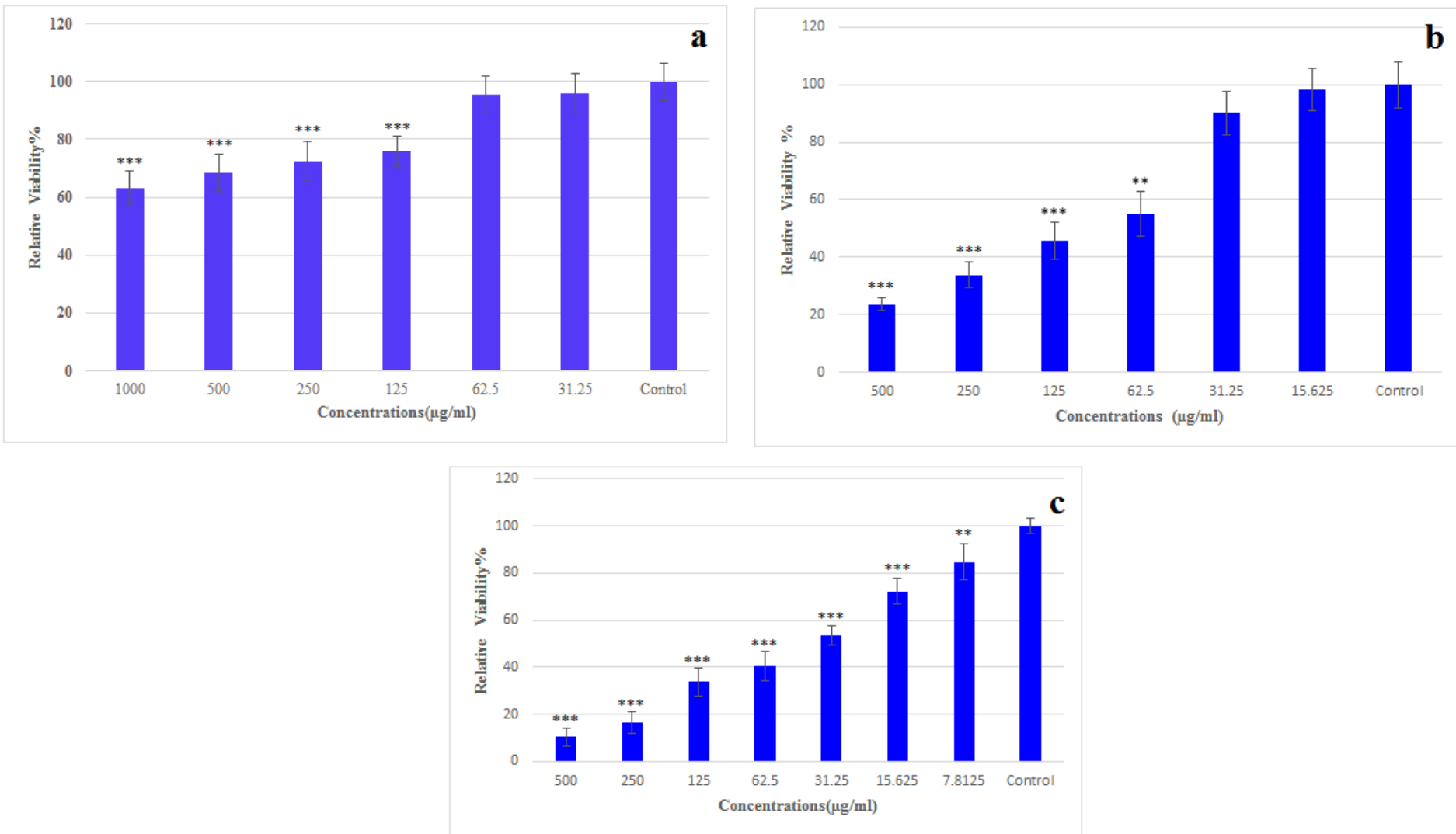

Figure 7

Cytotoxic effect of CuO@Glu/TSC NPs on a) normal fibroblast cells, and b) AGS cell, and c) cytotoxicity of cisplatin for AGS cells

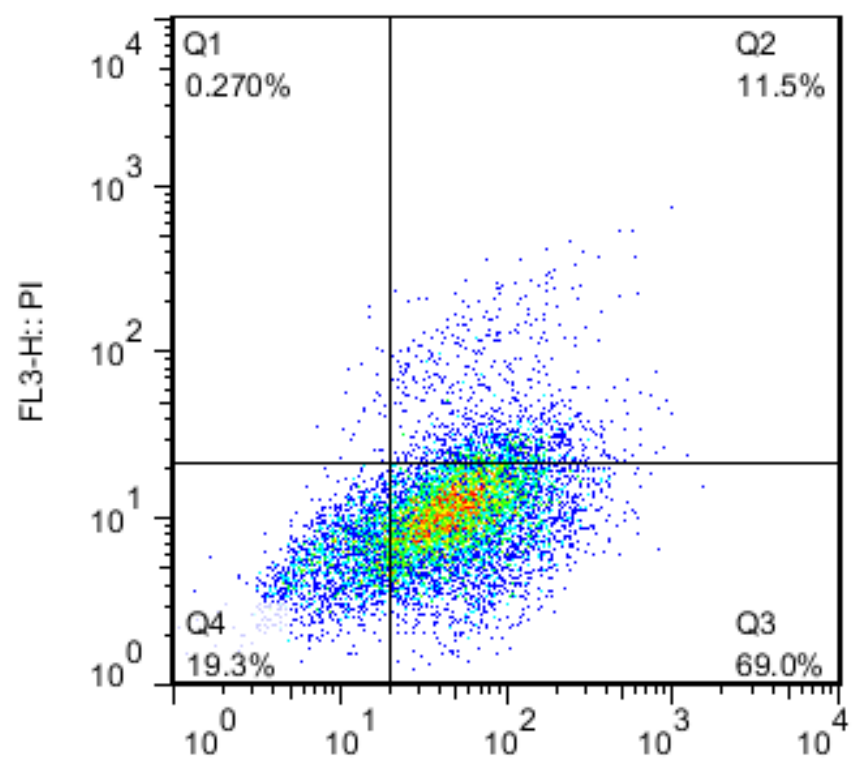

FL1-H:: Annexin V-FITC

(a)

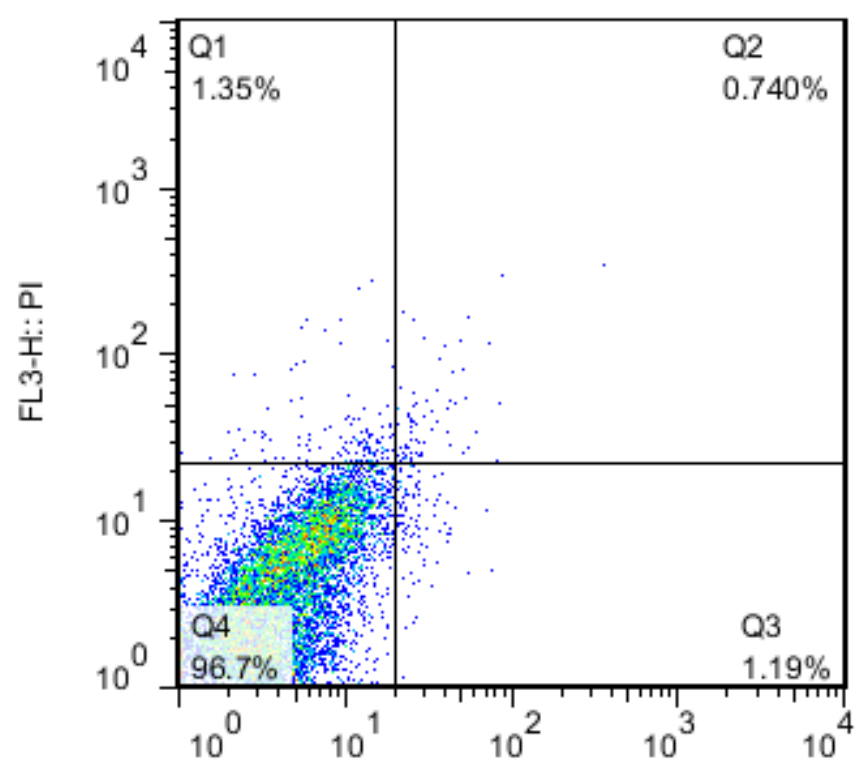

FL1-H:: Annexin V-FITC

(b) 
Figure 8

Flow-cytometry analysis of a) CuO@Glu/TSC treated, and (b) control AGS cells

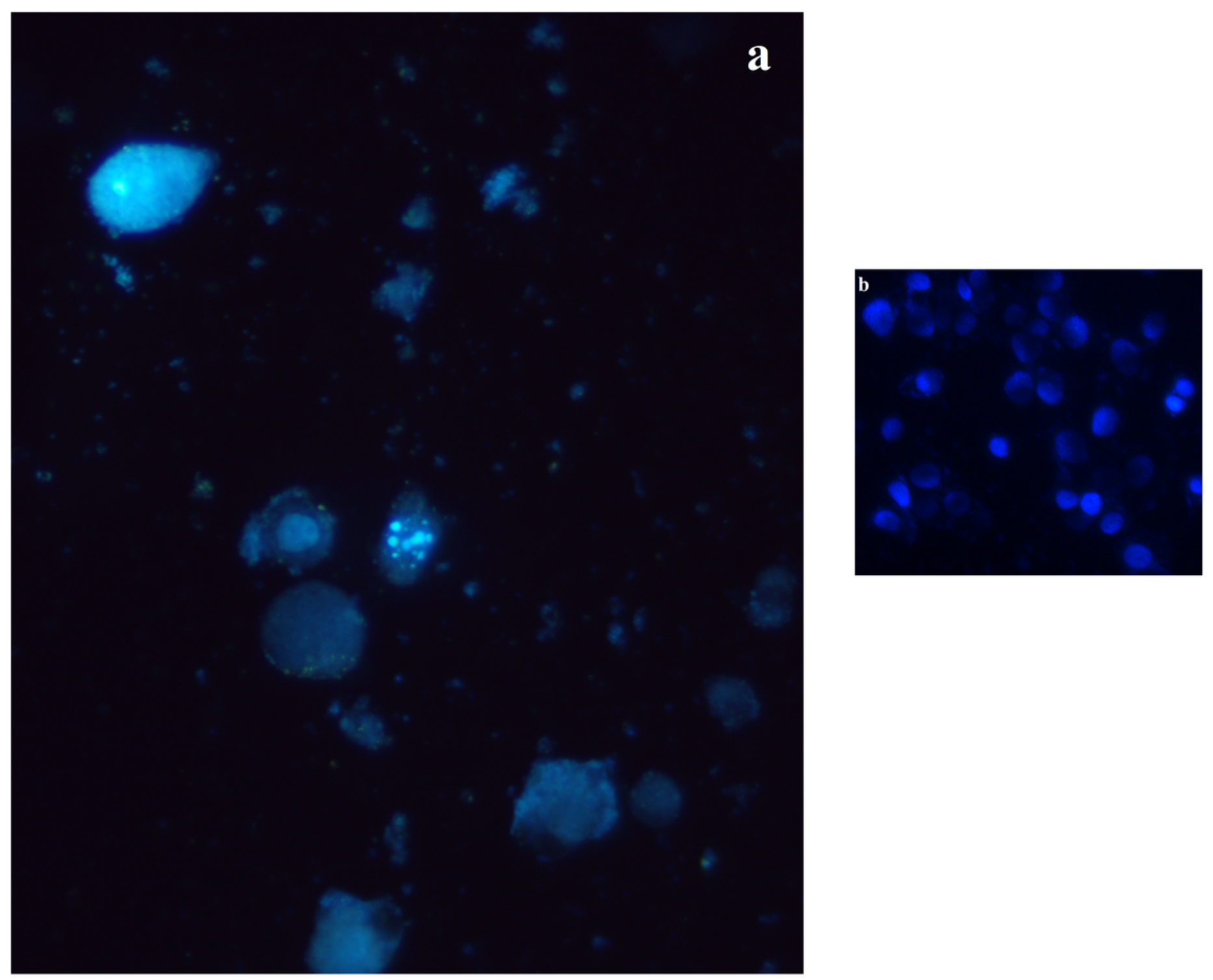

Figure 9

Hoechst staining of a) CuO@Glu/TSC treated, and b) control AGS cells. The apoptosis changes induced byCuO@Glu/TSC could be observed in the NPs treated cells. 


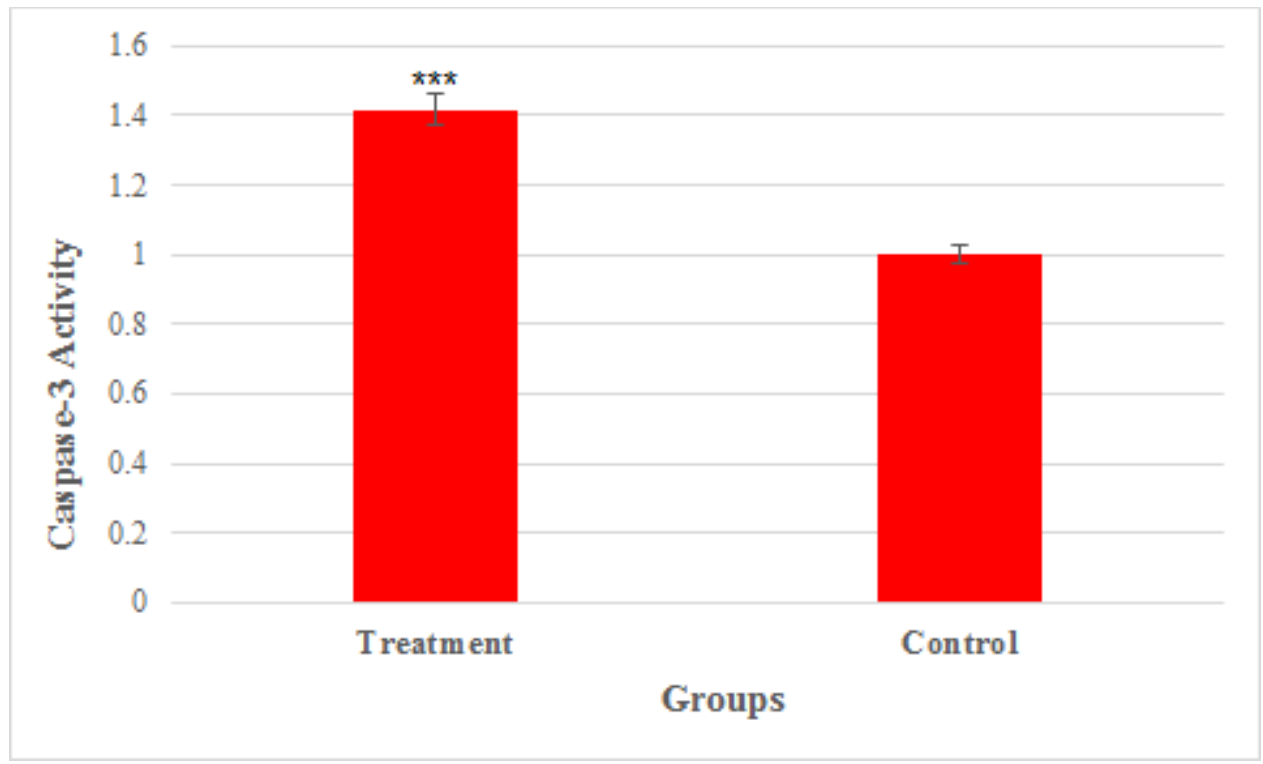

Figure 10

Caspase-3 activity among CuO@Glu/TSC treated and control AGS cells 